\title{
ON A FUNCTIONAL CALCULUS FOR DECOMPOSABLE OPERATORS AND APPLICATIONS TO NORMAL, OPERATOR-VALUED FUNCTIONS $\left({ }^{1}\right)$
}

\author{
BY
}

\section{FRANK GILFEATHER}

\begin{abstract}
Whenever $A=\int_{\boldsymbol{\Lambda}} \oplus A(\boldsymbol{\lambda}) \mu(d \boldsymbol{\lambda})$ is a decomposable operator on a direct integral $H=\int_{\boldsymbol{\Lambda}} \oplus H(\lambda) \mu(d \lambda)$ of Hilbert spaces and $f$ is a function analytic on a neighborhood of $\sigma(A)$, then we obtain that $f(A(\lambda))$ is defined almost everywhere and $f(A)(\lambda)=f(A(\lambda))$ almost everywhere. This relationship is used to study operators $A$, on a separable Hilbert space, for which some analytic function $A$ is a normal operator. Two main results are obtained. Let $f$ be an analytic function on a neighborhood of the spectrum of an operator $A$. If $f^{\prime \prime}(z) \neq 0$ for all $z$ in the spectrum of $A$ and if $f(A)$ is a normal operator, then $A$ is similar to a binormal operator. It is known that a binormal operator is unitarily equivalent to the direct sum of a normal and a two by two matrix of commuting normal operators. As above if $f(A)$ is normal and in addition, $f(z)-\zeta_{0}$ has at most two roots counted to their multiplicity for each $\zeta_{0}$ in the spectrum of $N$, then $A$ is a binormal operator.
\end{abstract}

In this paper, all spaces are complex separable Hilbert spaces and all operators on them are bounded. Various authors have investigated the structure of $A$ whenever a particular function of $A$ is normal. A. Brown [3] studied binormal operators which included operators satisfying $A^{2}=\lambda I$. He gave a structure theorem for binormal operators; these results have more recently been investigated in [2], and [15]. J. Stampfli has shown that if $A^{n}$ is normal and $A$ is invertible, then $A$ is similar to a normal operator; subsequently, S. Foguel and C. Apostol have considerably generalized this result ([1], [10], [12], [17]). In this paper, we generalize these results and show that it is possible to approach these and similar problems through reduction theory of operators. Questions, concerning the structure of operators, such as those investigated above will be reduced to questions about algebraic operators, which can then be handled with more elementary operator theory methods. This approach seems new and essentially different from previous works.

Received by the editors February 15, 1972.

AMS (MOS) subject classifications (1970). Primary 47A60, 47A65, 47B15, 46L10; Secondary 47B 40.

Key words and phrases. Normal operator, binormal operator, $W^{*}$ algebra, direct integral reduction of a $W^{*}$ algebra, functional calculus for operators.

(1) This work was partially supported by the University of Hawaii Research Council under a grant from the National Science Foundation. 
Let $\mathcal{F}(A)$ be the algebra of all functions $f$ which are analytic on some neighborhood of $\sigma(A)$. In $\S 1$, we relate the usual functional calculus for the functions in $\mathcal{F}(A)$, where $A=\int_{\boldsymbol{\Lambda}} \bigoplus A(\lambda) d \mu(\lambda)$ is a decomposable operator on a direct integral of Hilbert spaces (defined below), to the functional calculus on the functions in $\mathcal{F}(A(\lambda))$. Our main result in $\S_{1}$ is a Fubini type theorem which states that whenever $f \in \mathfrak{F}(A)$, then $f \in \mathfrak{F}(A(\lambda)) \mu$-a.e. and $f(A)(\lambda)=f(A(\lambda)) \mu$-a.e. As a necessary step in the study of the functional calculus on $\mathcal{F}(A)$, we obtain a new spectral inclusion for decomposable operators. Finally we extend the calculus on $\mathcal{F}(A)$ to an algebra $\mathcal{F}_{0}(A)$, containing $\mathcal{F}(A)$, where $\mathcal{F}_{0}(A)$ consists of certain analytic functions and is defined in terms of the decomposition of $A$.

In $\$ 2$, we give several applications of the functional calculus presented in \$1. C. Apostol has shown that $A$ is similar to a normal operator whenever there is an $f \in \mathcal{F}(A)$ with $f^{\prime}(z) \neq 0$ for $z \in \sigma(A)$ and $f(A)$ is a scalar operator in the sense of $\mathrm{N}$. Dunford ([1], [8]). Thus it is possible to completely determine the structure of $A$ whenever there exists an $f \in \mathcal{F}(A)$ with $f^{\prime}(z) \neq 0$, for $z \in \sigma(A)$, and $f(A)$ is normal. Using reduction theory, we show that it is possible to generalize C. Apostol's theorem to $f^{\prime \prime}(z) \neq 0$, if we use the structure theory of binormal operators to determine the operator $A$. Arlen Brown has introduced the class of binormal operators on Hilbert space, and has characterized them as those operators which are unitarily equivalent to the direct sum of a normal operator and a two by two matrix of commuting normal operators [3]. We show that if there exists an $f \epsilon$ $\mathcal{F}(A)$ for which $f(A)$ is a normal operator and where $f^{\prime \prime}(z) \neq 0$, for $z \in \sigma(A)$, then $A$ is similar to a binormal operator. The complete structure and unitary invariants of binormal operators are known and have been given by A. Brown [3] and more recently by $\mathrm{H}$. Radjavi and $\mathrm{P}$. Rosenthal [15].

Whenever the function $f \in \mathcal{F}(A)$ is one to one on a neighborhood of $\sigma(A)$, then it is clear that $f^{-1} \in \mathcal{F}(f(A))$. Thus if $f(A)$ is normal, it follows that $f^{-1}(f(A))$ $=A$ and therefore $A$ is normal. Thus we may replace the similarity of $A$ to a normal operator with the observation that $A$ is normal. We can generalize this easy result in the normal case to a theorem which includes several known results in the binormal case (cf. [2], [3], [15]). Let $f \in \mathcal{F}(A)$, for which the function of $z, f(z)-\zeta$, has at most two roots counted to their multiplicity, for each $\zeta \epsilon$ $f(\sigma(A))$. We shall show that if $f(A)$ is normal, then $A$ is binormal. Finally, our results shall be stated more generally for functions in the algebra $\mathcal{F}_{0}(A)$, which for every $A$ includes the functions in $\mathcal{F}(A)$.

For the details of the direct integral decomposition of a von Neumann algebra, we refer to [16], however, we shall introduce some basic notations and results here. Let $\mu$ be finite positive regular measure defined on the Borel sets of a separable metric space $\Lambda$, and let $e_{n}, 1 \leq n \leq \infty$, be a collection of disjoint Borel sets of $\Lambda$ with union $\Lambda$. Let $H_{1} \subseteq H_{2} \subseteq \cdots \subseteq H_{\infty}$ be a sequence of Hilbert spaces, with $H_{n}$ having dimension $n$ and $H_{\infty}$ being separable. By 


$$
H=\int_{\Lambda} \bigoplus H(\lambda) \mu(d \lambda)
$$

we shall denote the space of weakly $\mu$-measurable functions from $\Lambda$ into $H_{\infty}$ such that $f(\lambda) \in H_{n}$, if $\lambda \epsilon e_{n}$, and $\int_{\boldsymbol{\Lambda}}|f(\lambda)|^{2} \mu(d \lambda)<\infty$. The space $H$ is a Hilbert space, and we shall denote the element $f \in H$ determined by the vector valued function $f(\lambda)$ as $\int_{\boldsymbol{\Lambda}} \bigoplus f(\lambda) \mu(d \lambda)$.

An operator $A$ on $H$ is said to be decomposable if there exists a $\mu$-measurable operator valued function $A(\lambda)$ so that $(A f)(\lambda)=A(\lambda) f(\lambda)$ for $f \in H$. The operator $A$ is denoted by

$$
A=\int_{\Lambda} \bigoplus A(\lambda) \mu(d \lambda)
$$

Furthermore, every von Neumann algebra $\mathscr{A}$ on a separable space is spatially isomorphic to an algebra of decomposable operators on a direct integral of Hilbert spaces, such that the von Neumann algebra $\mathscr{U}(\lambda)$ generated by $\{A(\lambda)\}$, where $A \in$ $\mathcal{U}$, is a factor $\mu$-a.e. Finally we use the fact that if $A=\int_{\mathbf{\Lambda}} \bigoplus A(\lambda) d \mu(\lambda)$ generates $\mathcal{U}$, then $A(\lambda)$ generates the von Neumann algebra $\mathfrak{U}(\lambda) \mu$-a.e. Whenever in our use of this decomposition, there is no confusion over the space $\Lambda$, we shall suppress it.

If $A$ is an operator, we shall denote by $R(A), R(A)^{\prime}$ and $Z(A)$, respectively, the von Neumann algebra generated by $A$, the commutant of $R(A)$ and the center of $R(A)$. N. Suzuki has introduced the notion of a primary operator. One calls an operator $A$ primary, in case $R(A)$ is a factor, i.e. $Z(A)$ is just the scalar multiples of the identity. Let $A$ be defined on a separable Hilbert space and let $H=$ $\int_{\mathbf{\Lambda}} \bigoplus H(\lambda) \mu(d \lambda)$ be the direct integral decomposition of $H$ related to $R(A)$ for which the algebra $R(A)(\lambda)$ is a factor $\mu$-a.e., then this decomposition is unique in the sense of $\left[16\right.$, I.6]. Thus the operator $A$ is decomposed as $A=\int \bigoplus A(\lambda) \mu(d \lambda)$, where $A(\lambda)$ is primary $\mu$-a.e., and we shall refer to this particular decomposition as the primary decomposition of $A$. Finally, for convenience, we shall use the symbols $A \leftrightarrow B$ to denote the fact that $A B=B A$ and we shall let $R(\zeta ; A)$ denote $(\zeta I-A)^{-1}$.

1. A functional calculus for a decomposable operator. In the set of decomposable operators with respect to the direct integral of Hilbert spaces $H=$ $\int \bigoplus H(\lambda)_{\mu}(d \lambda)$, it is easy to show certain operational identities. For example, if $A$ and $B$ are decomposable and $\alpha, \beta$ are scalars, then

(i) $\alpha A+\beta B$ is decomposable and $\alpha A+\beta B=\int \bigoplus \alpha A(\lambda)+\beta B(\lambda) \mu(d \lambda)$,

(ii) $A B$ is decomposable and $A B=\int \bigoplus A(\lambda) B(\lambda) \mu(d \lambda)$.

Hence if $p(z)$ is any polynomial, then $p(A)$ is decomposable and $p(A)(\lambda)=$ $p(A(\lambda)) \mu$-a.e. Let $f \in \mathcal{F}(A)$, the algebra of functions which are analytic on a domain containing $\sigma(A)$, then $f(A)$ is defined by

$$
f(A)=\frac{1}{2 \pi i} \int_{B} f(z) R(z ; A) d z
$$


where $B$ consists of a finite number of rectifiable Jordon curves orientated in the usual way (cf. [8, VII.3)]. Also for each $\lambda$, there exists the same functional calculus for the functions $\mathscr{F}(A(\lambda))$. In this section we show the relation between the functional calculus on $\mathfrak{F}(A)$ and $\mathcal{F}(A(\lambda))$ and thus extend the result, which is true for polynomials, to the analytic functions in $\mathcal{F}(A)$.

The main result of this section is Theorem 1, which contains a type of Fubini theorem for decomposable operators. Specifically, we shall show that whenever $f \epsilon$ $\mathfrak{F}(A)$, then $f \in \mathfrak{F}(A(\lambda)) \mu$-a.e. and moreover, $f(A)$ is decomposable with $f(A)(\lambda)=$ $f(A(\lambda))$ almost everywhere, that is,

$$
\int_{B} f(\zeta) \int_{\Lambda} \oplus R(\zeta, A(\lambda)) \mu(d \lambda) d \zeta=\int_{\Lambda} \oplus \int_{B} f(\zeta) R(\zeta, A(\lambda)) d \zeta \mu(d \lambda)
$$

It is easy to construct a direct sum of operators $A=\sum_{i=1}^{\infty} \bigoplus A_{i}$, for which $\overline{\bigcup_{i=1}^{\infty} \sigma(A)}=\{0\}$ and $\sigma(A)=\{\lambda|| \lambda \mid \leq 1\}$, (cf. remark following Proposition 2). However, it is always the case that $\overline{\bigcup_{i=1}^{\infty} \sigma\left(A_{i}\right)} \subset \sigma(A)$ and, under certain uniform growth conditions on the resolvents of $A_{i}$, we have that $\overline{\bigcup_{i=1}^{\infty} \sigma\left(A_{i}\right)}=\sigma(A)$. Since a decomposable operator on a direct integral of Hilbert spaces is a generalization of a direct sum of operators on a direct sum of Hilbert spaces, we may expect similar spectral relations for decomposable operators. We shall give these analogous relations in Propositions 1 and 2 of this section.

Recently T. R. Chow has given an extensive account of the theory of decomposable spectral operators [5]. In his work, he proves the following spectral inclusion for a decomposable operator $A=\int_{\boldsymbol{\Lambda}} \bigoplus A(\lambda) \mu(d \lambda)$ on $H(\lambda) \mu(d \lambda)$ :

$$
\left\{\bigcap_{\delta} \overline{\bigcup_{\lambda \in \delta} \sigma(A(\lambda))}: \mu(\delta)=\mu(\Lambda)\right\} \subseteq \sigma(A),
$$

(cf. [5] and [7]). We shall show that, in fact, a stronger inclusion holds; in Proposition 1 we shall prove that $\sigma(A(\lambda)) \subset \sigma(A) \mu$-a.e. To prove our inclusion, we first give a lemma which also appears in [4].

Lemma 1. If $A=\int_{\boldsymbol{\Lambda}} \bigoplus A(\lambda) \mu(d \lambda)$ on $H=\int_{\boldsymbol{\Lambda}} \bigoplus H(\lambda) \mu(d \mu)$, then $r(A) \geq$ ess $\sup r(A(\lambda))$, where $r^{(\cdot)}$ is the spectral radius.

Proof. Since $\|A\|=$ ess sup $\|A(\lambda)\|$ and hence $\left\|A^{n}\right\|=$ ess sup $\left\|A^{n}(\lambda)\right\|$, there are sets of measure zero, $\eta_{n}$, so that $\left\|A^{n}\right\| \geq\left\|A^{n}(\lambda)\right\|=\left\|A(\lambda)^{n}\right\|$ for $\lambda \notin \eta_{n}$. Let $\eta=$ $\bigcup_{n=1}^{\infty} \eta_{n}$, then $\eta$ is a set of measure zero and, for $\lambda \notin \eta,\left\|A^{n}\right\| \geq\left\|A(\lambda)^{n}\right\|$ for $n=$ $1,2, \cdots$. Therefore $\left\|A^{n}\right\|^{1 / n} \geq\left\|A(\lambda)^{n}\right\|^{1 / n} \mu$-a.e. and $r(A) \geq r(A(\lambda)) \mu$-a.e.

Now we state and prove the spectral inclusion mentioned above.

Proposition 1. If $A=\int_{\boldsymbol{\Lambda}} \bigoplus A(\lambda) \mu(d \lambda)$ is a decomposable operator on the space $H=\int_{\mathbf{\Lambda}} \bigoplus H(\lambda) \mu(d \lambda)$, then $\sigma(A(\lambda)) \subset \sigma(A)$ almost everywhere. 
Proof. We shall argue by contradiction. Let the measure of $X=\{\lambda \mid \sigma(A(\lambda)) \cap$ $\rho(A) \neq \varnothing$ be greater than zero $(\rho(A)$ is the resolvent set of $A)$. Since every open set in the complex plane is the countable union of open spheres, there is a sphere $S_{r, \lambda_{0}}$ in $\rho(A)$ with radius $r$ and center $\lambda_{0}$ so that the measure of $S_{r, \lambda_{0}} \cap X$ is greater than zero. If we replace the operator $A$ by $B=r^{-1}\left(A-\lambda_{0} I\right)$, then by the Spectral Mapping Theorem, we have that $\sigma(B)$ lies outside the unit disk $D$. We now have $B=\int_{\Lambda} \bigoplus B(\lambda) \mu(d \lambda)$, where $B(\lambda)=r^{-1}\left(A(\lambda)-\lambda_{0}\right)$, and the measure of $\{\lambda \mid \sigma(B(\lambda)) \cap D \neq \varnothing\}$ is greater than zero. Finally, let us consider $B^{-1}$; the spectrum of $B^{-1}$ is contained in $D$ and hence $r\left(B^{-1}\right) \leq 1$; however, for a set of positive measure, $r\left(B(\lambda)^{-1}\right)>1$. This contradicts Lemma 1 and proves the proposition.

Let $\delta$ be a set of measure zero so that $\sigma(A(\lambda)) \subset \sigma(A)$ for each $\lambda \notin \delta$ and assume that the functions $\zeta \rightarrow\left\|(\zeta I(\lambda)-\Lambda(\lambda))^{-1}\right\|$ are essentially bounded, whenever $\zeta \notin \overline{\bigcup_{\lambda d \delta} \sigma(\Lambda(\lambda))}$. Then the measurable operator valued function $R(\zeta ; \Lambda(\lambda))$ is essentially bounded on $\Lambda$ and hence $(\zeta I-\Lambda)^{-1}$ exists. Therefore the complement of $\overline{\bigcup_{\lambda \notin \delta} \sigma(\Lambda(\lambda))}$ is contained in the resolvent of $\Lambda$ and thus $\overline{\bigcup_{\lambda \notin \delta} \sigma(\Lambda(\lambda))}$ $=\sigma(A)$. In particular if the resolvent function of $A(\lambda)$ has a reasonable uniform growth condition for almost all $\lambda$, then we can determine a spectral equality. We say that the resolvent of $A$ has growth rate of order $\alpha$ with constant $K$, if $\|R(\zeta ; \Lambda)\| \leq K d(\zeta, \sigma(\Lambda))^{-\alpha}$, where we write $d(\zeta, S)$ for the distance between $\zeta$ and the set $S$.

Proposition 2. Let $A$ be the decomposable operator $A=\int_{\mathbf{\Lambda}} \bigoplus A(\lambda) d \mu(\lambda)$. If, for almost all $\lambda$, the resolvent of $\Lambda(\lambda)$ bas growth rate of order $\alpha \geq 1$ and constant $K$, then there is a set $\delta$ of measure zero, sucb that $\sigma(A)=\overline{\bigcup_{\lambda \notin \Lambda-\delta} \sigma(\Lambda(\lambda))}$ and the resolvent of $\Lambda$ satisfies a growth rate of order a with constant $K$.

Proof. By Proposition 1, there is a set $\delta_{1}$ of measure zero such that if $\lambda \epsilon$ $\Lambda-\delta_{1}$, then $\sigma(\Lambda(\lambda)) \subset \sigma(A)$. By the hypothesis there is a set $\delta_{2}$ of measure zero such that $R(\zeta ; \Lambda(\lambda))$ has a growth rate of order $a$ and constant $K$ for $\lambda \notin \Lambda-\delta_{2}$. Let $\delta=\delta_{1} \cup \delta_{2}$ and $S=\overline{\bigcup_{\lambda / \delta} \sigma(\Lambda(\lambda))}$; thus $S(\sigma(\Lambda)$ and if $\lambda \epsilon \Lambda-\delta$, then $\|R(\zeta ; \Lambda(\lambda))\| \leq K d(\zeta, \Lambda(\lambda))^{-\alpha} \leq K d(\zeta, S)^{-\alpha}$. Since $K d(\zeta, S)^{-\alpha}$ is independent of $\lambda$, we see by the paragraph preceding Proposition 2, that $\sigma(\Lambda)=\overline{\bigcup_{\lambda f \delta} \Lambda(\lambda)}$. Furthermore

$$
\begin{aligned}
\|R(\zeta, \Lambda)\| & - \text { ess sup }\|R(\zeta, \Lambda(\lambda))\| \\
& \leq \text { ess sup } K d(\zeta, \Lambda(\lambda))^{-a} \leftrightharpoons K d(\zeta, \sigma(\Lambda))^{-a} .
\end{aligned}
$$

Remark. As one would expect, the converse of Proposition 2 is not true even for an infinite direct sum of operators. Let $\Lambda_{n}$ be the nilpotent operator defined on an $n$-dimensional Hilbert space $H_{n}$ which is given by the matrix 


$$
\left(\begin{array}{cccccc}
0 & 0 & \cdot & \cdot & \cdot & 0 \\
1 & 0 & \cdot & \cdot & \cdot & 0 \\
0 & 1 & 0 & \cdot & \cdot & 0 \\
\vdots & 0 & & 0 & 0 & 0 \\
0 & \cdot & 0 & 0 & 0
\end{array}\right)
$$

If $A=\Sigma_{i=1}^{\infty} \bigoplus A_{i}$ on the Hilbert space $H=\Sigma_{i=1}^{\infty} \bigoplus H_{i}$, then one can also represent $A$ as a unilateral weighted shift on an infinite dimensional space with weights $(0,1,0,1,1,0,1,1,1,0, \cdots)$. Thus $\sigma(A)=\bar{D}=\{\zeta|| \zeta \mid \leq 1\}$; however for each $n, \sigma\left(A_{n}\right)=\{0\}$. Furthermore, if $|\zeta|>1$, then $\left\|(\zeta-A)^{-1}\right\| \leq(|\zeta|-1)^{-1}$; hence the resolvent growth rate of $A$ is 1 . On the other hand, if we calculate $R\left(\zeta_{;} A_{n}\right)$, we see that it has a pole of order $n$ at zero. Therefore the resolvents of $A_{n}$ have no uniform growth rate even though $A$ does (cf. [11, p. 243]).

Now we present the main theorem of this section. As we have mentioned, it extends the operational calculus for polynomial functions of a decomposable operator to the functions in $\mathcal{F}(A)$ and thus it gives the relation between the functional calculus on $\mathcal{F}(A)$ and on $\mathcal{F}(A(\lambda))$.

Theorem 1. Let $A$ be a decomposable operator on $H=\int_{\boldsymbol{\Lambda}} \bigoplus H(\lambda) d \mu(\lambda)$. Let $f \in \mathcal{F}(A)$, then $f \in \mathcal{F}(A(\lambda)) \mu$-a.e. and ess sup $\|f(A(\lambda))\|<\infty$; moreover, $f(A)$ is decomposable, and $f(A)(\lambda)=f(A(\lambda)) \mu$-a.e. Whenever $f, g \in \mathcal{F}(A)$ and $\alpha, \beta$ are scalar, then

(a) $(\alpha f+\beta g)(A)$ is decomposable and $(\alpha f+\beta g)(A \lambda))=\alpha f(A(\lambda))+\beta g(A(\lambda))$ $\mu$-a.e.,

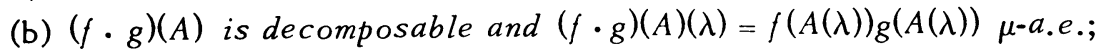
if $g \in \mathcal{F}(A)$ and $f \in \mathcal{F}(g(A))$, then

(c) $(f \circ g)(A)$ is decomposable and $(f \circ g)(A)(\lambda)=f(g(A(\lambda)))$-a.e.

Proof. Statements (a), (b), and (c) all follow from the usual functional calculus on $\mathcal{F}(A)$ once we show that, for $f \in \mathcal{F}(A), f(A)$ is decomposable and $f(A)(\lambda)=$ $f(A(\lambda)) \mu$-a.e. That $f(A)$ is decomposable for any $f \in \mathcal{F}(A)$ follows because $f(A)$ $\epsilon R(A)$ and every operator in $R(A)$ is decomposable.

Let $f \in \mathfrak{F}(A)$, that is, $f$ is an analytic function on a domain $U$ containing $\sigma(A)$ so that $B$, the boundary of the domain $U$, consists of a finite number of rectifiable Jordon curves. Since $B$ is compact there is a constant $M$, independent of $\zeta \in B$, so that $\|R(\zeta ; A)\| \leq M$. Let $\left\{\zeta_{n}\right\}$ be a dense set in $B$; then by the condition for the existence of $R\left(\zeta_{n} ; A\right)$, for each $\zeta_{n},\|R(\zeta ; A(\lambda))\| \leq M \mu$-a.e. Let $\eta_{n}$ be a set of measure zero, for which $\lambda \in \Lambda-\eta_{n}$ implies that $\left\|R\left(\zeta_{n}, A(\lambda)\right)\right\| \leq M$. If $\eta=$ $\bigcup \eta_{n}$, then $\eta$ is a set of $\mu$ measure zero and $\lambda \in \Lambda-\eta$ implies that $\left\|R\left(\zeta_{n}, A(\lambda)\right)\right\|$ $\leq M$ for all $n$. Since $\left\{\zeta_{n}\right\}$ is dense in $B$ and $\|\cdot\|$ is continuous, it follows that $\bar{\lambda} \rightarrow\|R(\zeta ; A(\lambda))\|$ is $\mu$-essentially bounded by $M$ uniformly on $B$. Let $\left\{p_{n}(A)\right\}$ be a sequence of partial sums of $f(A)$ converging uniformly to $f$ of the form: 


$$
p_{n}(A)=\frac{1}{2 \pi i} \sum_{i=1}^{n_{k}} f\left(z_{n, i}^{\prime}\right) R\left(z_{n, i}^{\prime} ; A\right)\left(z_{n, i}-z_{n, i-1}\right),
$$

where moreover we may take $\max \left|z_{n, i}-z_{n, i-1}\right|<1 / n$. For each $n$ there is a set of measure zero $\eta_{n}^{\prime}$, where if $\lambda \in \Lambda_{-}-\eta_{n}^{\prime}$, then $p_{n}(A)(\lambda)=p_{n}(A(\lambda))$ and $\| p_{n}(A)(\lambda)-$ $f(A)(\lambda)\|\leq\| p_{n}(A)-f(A) \|$. Let $\eta^{\prime}=\bigcup_{n=1}^{\infty} \eta_{n}^{\prime}$, for $\lambda \in \Lambda-\eta^{\prime}$ and any $n$, we have that $\left\|p_{n}(A(\lambda))-f(A)(\lambda)\right\|=\left\|p_{n}(A)(\lambda)-f(A)(\lambda)\right\| \leq\left\|p_{n}(A)-f(A)\right\|$. Since $p_{n}(A)$ converges uniformly to $f(A)$, then for $\lambda \in \Lambda-\eta^{\prime}, p_{n}(A(\lambda))$ converges to $f(A)(\lambda)$.

Let $\eta^{\prime \prime}$ be a set of $\mu$ measure zero determined by Proposition 1 so that $\sigma(A(\lambda)) \subset \sigma(A)$, whenever $\lambda \in \Lambda-\eta^{\prime \prime}$. Finally if $\delta=\eta \cup \eta^{\prime} \cup \eta^{\prime \prime}$, then for $\lambda \in \Lambda$ $\delta$ the operator $f(A(\lambda))$ exists; $p_{n}(A(\lambda))$ converges to $f(A)(\lambda)$; and $\|R(\zeta ; A(\lambda))\| \leq$ $\|R(\zeta ; A)\|$. By the definition of the Radon-Stieltjes integral, we have that $f(A)(\lambda)=$ $f(A(\lambda))$ for $\lambda \in \Lambda-\delta$.

The converse of Theorem 1 does not follow as it is possible to construct a decomposable operator $A$ and an analytic function $f$ on a domain containing $\bigcup_{\lambda \in \boldsymbol{\Lambda}} \sigma(A(\lambda))$, i.e. $f \in \mathcal{F}(A(\lambda))$, for which ess sup $\|f(A(\lambda))\|<\infty$ and yet $f \notin \mathcal{F}(A)$. We shall give such an example following Lemma 2. This situation arises since $f$ may not have an analytic extension to a domain containing $\sigma(A)$, even though $f$ is analytic on a domain containing $\overline{\bigcup_{\lambda \in \Lambda} \sigma(A(\lambda))}$. However with the hypothesis on $A$ given in Proposition 2, it is always possible to define such an extension.

Proposition 3. Let $A$ be a decomposable operator on $H=\int_{\boldsymbol{\Lambda}} \bigoplus H(\lambda) \mu(d \lambda)$ and $\delta$ a Borel set with $\mu(\Lambda-\delta)=0$, for which $\overline{\bigcup_{\lambda \in \delta} \sigma(A(\lambda))}=\sigma(A)$. Let $f_{\lambda}$ be defined on a domain $\mathfrak{T}_{\boldsymbol{\lambda}} \supset \sigma(A(\lambda))$, so that $f_{\boldsymbol{\lambda}} \in \mathcal{F}(A(\lambda))$ and $f_{\boldsymbol{\lambda}}=f_{\alpha}$ on $\mathfrak{T}_{\boldsymbol{\lambda}} \cap \mathfrak{D}_{\alpha}$; then there exists an $f \in \mathcal{F}(A)$ with $f \mid \mathscr{D}_{\lambda}=\hat{f}_{\lambda}$.

Proof. If we set $f(\zeta)=f_{\lambda}(\zeta)$ for $\zeta \in \mathscr{D}_{\lambda}$, then $f$ is an analytic function on $\mathfrak{D}=\bigcup_{\lambda \in \delta} \mathscr{D}_{\lambda}$. Since $\mathfrak{D} \supset \sigma(A)$, we have $f \in \mathcal{F}(A)$ and clearly $f \mid \mathscr{D}_{\lambda}=f_{\lambda}$.

Now let us consider a fixed direct a fixed direct integral of Hilbert spaces, $H=\int_{\boldsymbol{\Lambda}} \bigoplus H(\lambda) \mu(d \lambda)$ and a decomposable operator $A$ on $H$. In case there exists a Borel set $\delta \subset \Lambda$ with $\mu(\Lambda-\delta)=0$ and $S(\delta) \equiv \overline{\bigcup \sigma(A(\lambda))} \subset \sigma(A)$, then we may consider an analytic function on $S(\delta)$ instead of $\sigma(A)$ (it follows from Proposition 1 that $\sigma(A(\lambda)) \subset \sigma(A) \mu$-a.e. $)$. We shall then call $\bigcap\{S(\delta): \mu(\Lambda-\delta)=0\}$ the reduced spectrum of $A$ and denote it by $r_{0}(A)$. Next we use $r_{0}(A)$ to define a new class of functions of $A$, which contains $\mathcal{F}(A)$, and we shall show the spectral mapping property for them with respect to the reduced spectrum.

Let $f$ be an analytic function on a domain $\mathcal{T}$ containing $r_{0}(A)$. By a compactness argument, one can show that there exists a set $\delta$ with $\mu(\Lambda-\delta)=0$ for which $S(\delta) \subset \mathscr{T}$. Therefore $f \in \mathcal{F}(A(\lambda))$ almost everywhere, and we may therefore consider ess sup $\|f(A(\lambda))\|$ by considering $f(A(\lambda))=0$ for $\lambda \in \Lambda-\delta$. Let $\mathcal{F}_{0}(A)$ be the set of functions $f$, analytic in a neighborhood of $r_{0}(A)$ and with $\|f(A(\lambda))\| \mu$-essentially bounded. The operator valued function $\lambda \rightarrow f(A(\lambda))$, which is defined almost 
everywhere by the remark above, is measurable since it is the limit almost everywhere of measurable functions. The boundedness condition implies that $\int_{\boldsymbol{\Lambda}} \bigoplus f(A(\lambda))_{\mu}(d \lambda)$ defines a bounded operator in $R(A)$ which we shall call $f(A)$. The following spectral mapping theorem we state without proof as it requires only routine compactness arguments and is not used in the sequel.

Lemma 2. If $f \in \mathcal{F}_{0}(A)$, then $r_{0}(f(A))=f\left(r_{0}(A)\right)$.

It is easy to see that we have a functional calculus for the functions in $\mathcal{F}_{0}(A)$, which extends the functional calculus on $\mathscr{F}(A)$. In case $A$ is normal, it is clear from Propositions 2 and 3 that $\mathcal{F}_{0}(A)=\mathcal{F}(A)$. T. R. Chow has shown that whenever $A$ is a spectral operator on a Hilbert space, then $\sigma(A)=r_{0}(A)[5]$, hence for spectral operators, it is also true that $\mathfrak{F}_{0}(A)=\mathfrak{F}(A)$.

In general, $\mathcal{F}_{0}(A) \neq \mathcal{F}(A)$ as the following example illustrates. If $A=$ $\sum_{i=1}^{\infty} \bigoplus A_{i}$ is defined as in the example following Proposition 2, then we shall show that $\mathcal{F}(A) \subset H^{\infty} \subset \mathcal{F}_{0}(A)$. Let $f=\sum_{n=1}^{\infty} a_{n} z^{n}$ be a function in $H^{\infty}$; if $A_{n}$ is nilpotent of order $n$, then $f\left(A_{n}\right)=\sum_{i=1}^{n} a_{i} A_{n}^{i}$. For $0<b<1$, the function $g_{b}(z)=f(b z)$ is analytic on a neighborhood of the closed unit sphere. Hence by von Neumann's theorem

$$
\left\|f\left(b A_{n}\right)\right\|=\left\|g_{b}\left(A_{n}\right)\right\| \leq \sup _{\|z\| \leq 1}\left\|g_{b}(z)\right\| \leq\|f\|_{\infty}
$$

and thus

$$
\left\|f\left(A_{n}\right)\right\|=\left\|\sum_{i=1}^{n} a_{i} A_{n}^{i}\right\|=\lim _{b \rightarrow 1}\left\|\sum_{i=1}^{n} a_{i} b^{i} A_{n}^{i}\right\|=\lim _{b \rightarrow 1}\left\|f\left(b A_{n}\right)\right\| \leq\|f\|_{\infty} .
$$

Therefore $H^{\infty} \subset \mathcal{F}_{0}(A)$ and since $\sigma(A)=\{z|| z \mid \leq 1\}$, it follows that $\mathcal{F}(A) \neq \mathcal{F}_{0}(A)$. Finally we remark that $r_{0}(A)$ and consequently $\mathcal{F}_{0}(A)$ are dependent on the direct integral of Hilbert spaces used to define them. To avoid this dependence, we may require that in their definitions, the primary decomposition is used. In this case, Lemma 2 becomes much more difficult and its validity is not known by the author.

2. Roots of normal operator-valued analytic functions. J. Stampfli and S. Kurepa showed that if $A$ is an invertible operator and $A^{n}$ is normal, then $A$ is a scalar type operator, i.e. similar to a normal operator ([12], [17]). S. Foguel generalized this result by showing that if $p(A)$ is a scalar type operator and $p^{\prime}(z) \neq 0$ for $z \in \sigma(A)$, then $A$ is a scalar type operator [10]. In 1943, E. R. Lorch noted that whenever $\exp (A)=I$, then $A$ has a simple structure and is in fact a scalar type operator [13]. These results are all special cases of a theorem due to C. Apostol which we give below as Theorem 3. Our main theorem characterizes operators as 
similar to binormal operators whenever certain functions of them are normal. The results of this section shall be the direct analogues of the results cited above to the binormal case.

An operator $A$ is called binormal if whenever $\left\{A_{1}, \cdots, A_{4}\right\}$ belongs to the von Neumann algebra $R(A)$, then

$$
\sum_{\sigma}(\operatorname{sign} \sigma) A_{\sigma(1)} \cdots A_{\sigma(4)}=0
$$

where the sum is taken over all permutations $\sigma$ on $\{1, \cdots, 4\}$. A. Brown has characterized these operators, as mentioned in the introduction, and has given a complete set of unitary equivalences for them [3].

Theorem 2. Let $f \in \mathcal{F}_{0}(A)$, so that $f^{\prime \prime}(z) \neq 0$ for $z \in r_{0}(A)$, the reduced spectrum of $A$. If $f(A)$ is normal, then $A$ is similar to a binormal operator.

Proof. Let $A=\int_{\Lambda} \bigoplus A(\lambda) \mu(d \lambda)$ be the primary decomposition of $A$ on the space $H=\int_{\mathbf{\Lambda}} \bigoplus H(\lambda) \mu(d \lambda)$. If $f(A)=N$ is normal, then by the operational calculus for decomposable operators and the Putnam-Fugledge theorem, $N \in Z(A)$ and hence $N=\int_{\boldsymbol{\Lambda}} \bigoplus g(\lambda) I(\lambda) \mu(d \mu)$, where $g(\lambda)$ is an essentially bounded Borel function. Therefore $f(A(\lambda))-g(\lambda) I(\lambda)=f(A)(\lambda)-g(\lambda) I(\lambda)=0$ almost everywhere, as $f \epsilon$ $\mathcal{F}(A(\lambda))$ almost everywhere by Theorem 1 . If $\left\{r_{i}(\lambda)\right\}_{i=1}^{n}$ is the set of zeros of $f(z)-$ $g(\lambda)$ counted to their multiplicity in $\sigma(A(\lambda))$, then $f(z)-g(\lambda)=\Pi\left(z-r_{i}(\lambda)\right) b(z)$, where $b(z)$ is analytic and nonzero on $\sigma(A(\lambda))$. Since $b(z)^{-1} \in \mathcal{F}(A(\lambda))$, we conclude that

$$
0=(f(A(\lambda))-g(\lambda) I(\lambda)) b(A(\lambda))^{-1}=\prod_{i=1}^{n}\left(A(\lambda)-r_{i}(\lambda) I(\lambda)\right)
$$

The proof proceeds by showing that $n$ is independent of $\lambda$ and that the functions $r_{i}(\lambda)$ may be chosen so as to be measurable and thus determine the structure of $A(\lambda)$ from the identity

$$
\prod_{i=1}^{n}\left(A(\lambda)-r_{i}(\lambda) I(\lambda)\right)=0
$$

which holds almost everywhere.

From the functional calculus presented in $\$ 1$, we let $B$ be a closed curve bounding a domain which contains $r_{0}(A)$ and hence contains $\sigma(A(\lambda))$ almost everywhere. For each $\lambda$, 


$$
n(\lambda)=\frac{1}{2 \pi i} \int_{B} \frac{f^{\prime}(z)}{f(z)-g(\lambda)} d z
$$

and by Proposition $1, \sigma(N) \Delta\{g(\lambda) \mid \lambda \in \Lambda\}$ has zero measure; thus if we let a vary over the compact set $\sigma(N)$, then

$$
n(\alpha)=\int_{B} \frac{f^{\prime}(z)}{f(z)-\alpha} d z
$$

is bounded and therefore $n(\lambda)$ is essentially bounded. Let $N$ be the essential bound of $\{n(\lambda)\}$ and for the rest of the proof we shall only consider $\lambda$, with $n(\lambda) \leq$ $N$ and for which $f(A(\lambda))-g(\lambda) I(\lambda)=0$.

Next we wish to choose the functions $r_{i}(\lambda)$ with two additional properties, one of which is measurability. For the other, we want to have that for each $j$ there exists a $\delta>0$ independent of $j$ such that except for possibly one $j^{\prime} \neq j$, then $i \neq$ $j, j^{\prime}$ implies that $\left|r_{i}(\lambda)-r_{j}(\lambda)\right|>\delta$. We begin by assuming that there exists a sequence of triples $\left(\alpha_{n}, \beta_{n}, \gamma_{n}\right)$, with $\alpha_{n}, \beta_{n}, \gamma_{n}$ in $r_{0}(A)$, no two of which are equal, but each is a root of $f(z)-f\left(\alpha_{n}\right)$, and for which $\max \left\{\left|\alpha_{n}-\beta_{n}\right|,\left|\alpha_{n}-\gamma_{n}\right|\right\}$ $\rightarrow 0$. Since $r_{0}(A)$ is compact, there is a subsequence $\left\{n_{i}\right\}$, such that $\left\{\alpha_{n_{i}}\right\},\left\{\beta_{n_{i}}\right\}$ and $\left\{\gamma_{\dot{n}_{i}}\right\}$ all converge to $\alpha \in r_{0}(A)$. This contradicts the fact that $f^{\prime \prime}(\alpha)^{i} \neq 0$. Therefore there is a compact neighborhood $S$ of $r_{0}(A)$ and a $\delta^{\prime}>0$ such that if $\left\{a_{i}\right\}_{i=1}^{N}$ is the set of roots in $S$ of $f(z)-\zeta$, for some $\zeta$ in a compact neighborhood $\hat{S}$ of $\sigma(N)$, then $\left|a_{i}-a_{j}\right|>\delta^{\prime}$, except for possibly one $i \neq j$.

By utilizing the Local Mapping Theorem, we may cover $\hat{S}$ with a finite number of open sets $\left\{A_{j}\right\}_{j=1}^{k}$, so that the set $f^{-1}\left(A_{j}\right)$ consists of the union of disjoint open set $\left\{B_{j i}\right\} \leq N=1$, with the property that if $\alpha \in B_{j i}$ and $\beta \in B_{j k}, i \neq k$, then $\mid \alpha-$ $\beta \mid>\delta^{\prime} / 4=\delta$. For fixed $j$ the sets $B_{j i}$ are of two kinds, those on which $f$ is $1-1$ and those where $f^{\prime}$ has a zero. In the later case, we divide the $B_{j i}$ into two Borel sets whose union in $B_{j i}$ and so that, on each, $f$ is 1-1. By considering this division, we have exactly $N$ Borel sets $\left\{\Re_{j i}\right\}_{i=1}^{N}$ so that, on each, $f$ is $1-1$ and $\bigcup_{i=1}^{N} \mathfrak{B}_{j i}=f^{-1}\left(A_{j}\right) \cap S$. Furthermore, $|\alpha-\beta|>\delta$, if $\alpha \in B_{j i}$ and $\beta \in B_{j k}$, where $i \neq k$ except for possibly one pair $i$ and $k$.

With this topological choosing, we may now construct the Borel measurable functions $r_{i}$. Let $C_{j}$ be the Borel sets $g^{-1}\left(A_{j}\right)$,

$$
r_{i}=\sum_{j=1}^{N}\left[\left(f \mid B_{j i}\right)^{-1} \circ g\right] \chi_{C_{j}-\cup_{k<j} C_{k}}
$$

Since $\{\lambda \mid g(\lambda) \notin \hat{S}\}$ is a set of $\mu$ measure zero, we have $r_{i}$ defined a.e. and hence $r_{i}$ can be extended to $\Lambda$. Furthermore the functions $r_{i}$ possess the properties we desired of them. 
In the following discusion we shall fix $\lambda$ in order to exactly determine $A(\lambda)$. We shall construct algebraic projections $\left\{E_{i}(\lambda)\right\}_{i=1}^{N}$, so that $A(\lambda) E_{i}(\lambda)=E_{i}(\lambda) A(\lambda)$ and $A(\lambda) \mid E_{i}(\lambda) H(\lambda)$ is binormal. For each $m, 1, \cdots, N$, let $m^{\prime}$ be the integer other than $m$, if any, for which $\left|r_{m}(\lambda)-r_{m^{\prime}}(\lambda)\right|<\delta$. Define

$$
E_{m}(\lambda)=\frac{1}{2 \pi i} \int_{|\zeta|=\delta} R\left(\zeta+r_{m}(\lambda) ; A(\lambda)\right) d \zeta
$$

then $A(\lambda) E_{m}(\lambda)=E_{m}(\lambda) A(\lambda)$ and $\sigma(A(\lambda) / E(\lambda) H(\lambda))=\left\{r_{m}(\lambda), r_{m},(\lambda)\right\}$. From (1), we have that

$$
\left(A(\lambda)-r_{m}(\lambda) I(\lambda)\right)\left(A(\lambda)-r_{m^{\prime}}(\lambda) I(\lambda)\right) E_{m}(\lambda)=0 .
$$

Next we shall estimate the norm of $E_{m}(\lambda)$ and show that it is essentially bounded. Let $x \in H(\lambda)$ and $m, m^{\prime}$ be given with $\left|r_{m}(\lambda)-r_{m}{ }^{\prime}(\lambda)\right|<\delta$; then

$$
\begin{aligned}
& \left.\left\|E_{m}(\lambda) x\right\|=\| \prod_{i \neq m, m^{\prime}}\left[\left(A(\lambda)-r_{i}(\lambda) I(\lambda)\right)\right\rangle^{\prime} E_{m}(\lambda) H(\lambda)\right]^{-1}\left[A(\lambda)-r_{i}(\lambda) I(\lambda)\right] E_{m}(\lambda) x \| \\
& \quad \leq\left\|\prod_{i \neq m, m^{\prime}}\left[\left(A(\lambda)-r_{i}(\lambda) I(\lambda)\right) / E_{m}(\lambda) H(\lambda)\right]^{-1}\right\|\left\|\prod_{i \neq m, m^{\prime}}\left(A(\lambda)-r_{i}(\lambda) I(\lambda)\right) E_{m}(\lambda) x\right\|
\end{aligned}
$$

(4)

$$
\begin{aligned}
& =\left\|\prod_{i \neq m, m^{\prime}}\left[\left(A(\lambda)-r_{i}(\lambda) I(\lambda)\right) / E_{m}(\lambda) H(\lambda)\right]^{-1}\right\|\left\|\prod_{i \neq m, m^{\prime}}\left(A(\lambda)-r_{i}(\lambda) I(\lambda)\right) x\right\| \\
& \leq M M^{\prime}\|x\|,
\end{aligned}
$$

where the last two steps must be verified. For each $x \in H(\lambda), x=\sum_{i=1}^{k} E_{m_{i}} x$, where $m_{i} \in\{1, \ldots, N\}$ and only one of $j, j^{\prime}$ appears if $\left|r_{j}(\lambda)-r_{j^{\prime}}(\lambda)\right|<\delta$. Thus

$$
\begin{aligned}
\prod_{i \neq m, m^{\prime}}\left(A(\lambda)-r_{i} I(\lambda)\right) x & =\sum_{j=1}^{k} \prod_{i \neq m, m^{\prime}}\left(A(\lambda)-r_{i} I(\lambda)\right) E_{m_{j}}(\lambda) x \\
& =\prod_{i \neq m, m^{\prime}}\left(A(\lambda)-r_{i}(\lambda) I(\lambda)\right) E_{m}(\lambda) x \text { by }(3)
\end{aligned}
$$

and

$$
\left\|\prod_{i \neq m, m^{\prime}}\left(A(\lambda)-r_{i} I(\lambda)\right) x\right\| \leq M^{\prime}\|x\|, \quad \text { independent a.e. of } \lambda \text { and } m \text {. }
$$

Finally we must show that

$$
\left\|\prod_{i \neq m, m}\left[\left(A(\lambda)-r_{i}(\lambda) I(\lambda)\right) / E_{m}(\lambda) H(\lambda)\right]^{-1}\right\| \leq M .
$$


In order to do this we note that if, for all $i \neq m,\left|r_{i}(\lambda)-r_{m}(\lambda)\right|>\delta$, i.e., $m^{\prime}$ does not exist for $m$, then $A(\lambda) / E_{m}(\lambda) H(\lambda)=r_{m}(\lambda) I(\lambda) / E_{m}(\lambda) H(\lambda)$ and hence $\left\|\left[\left(A(\lambda)-r_{i}(\lambda) I(\lambda)\right) / E_{m}(\lambda) H(\lambda)\right]^{-1}\right\| \leq 1 /\left|r_{i}(\lambda)-r_{m}(\lambda)\right| \leq 1 / \delta$. If $m^{\prime}$ does exist for $m$, then

$$
\left[A(\lambda)-\zeta I(\lambda) / E_{m}(\lambda) H(\lambda)\right]^{-1}=\frac{\left[\left(r_{m}(\lambda)+r_{m}{ }^{\prime}(\lambda)-\zeta\right) I(\lambda)-A(\lambda)\right] / E_{m}(\lambda) H(\lambda)}{\left(\zeta-r_{m}(\lambda)\right)\left(\zeta-r_{m^{\prime}}(\lambda)\right)}
$$

and hence the norm of this operator is less than or equal to a constant $K /\left|\left(\zeta-r_{m}(\lambda)\right)\left(\zeta-r_{m},(\lambda)\right)\right|$ where $K$ is independent of $\lambda$ a.e. $m, 1 \leq m \leq N$, and $\zeta$, where $\zeta \in S$ is bounded. Now for $\zeta=r_{i}(\lambda)$, we have $\left\|\left[A(\lambda)-r_{i}(\lambda) I(\lambda) / E_{m}(\lambda) H(\lambda)\right]^{-1}\right\|$ $\leq K / \delta^{2}$, since $\left\{r_{i}(\lambda)\right\}$ are bounded almost everywhere. Since there are at most $N-$ 1 of the operators $\left(A(\lambda)-r_{i}(\lambda) I(\lambda)\right) / E_{m}(\lambda) H(\lambda)$ to consider, we have shown (5) and hence also verified the inequalities in (4).

Each of the projections $E_{m}(\cdot)$ as defined in (2) is the uniform limit of partial sums, each of which is measurable, and hence $E_{m}(\cdot)$ is measurable. Furthermore, we may modify the functions $E_{m}(\cdot)$ so that they remain measurable and so that $E_{m}{ }^{\prime}(\lambda)=0$, if $\left|r_{m}(\lambda)-r_{m},(\lambda)\right|<\delta$ and $m^{\prime}>m$. The new sequence of measurable projection valued functions has the following properties:

(i) $E_{i}(\lambda) E_{j}(\lambda)=\delta_{i j} E_{i}(\lambda)$,

(ii) $\sum_{i=1}^{N} E_{i}(\lambda)=I(\lambda)$, and

(iii) ess sup $\left\|E_{i}(\lambda)\right\| \leq M, i=1, \cdots, N$.

Next we define a new measurable function

$$
S(\lambda)=\left(\sum_{i=1}^{N} E_{i}(\lambda) E_{i}(\lambda)^{*}\right)^{1 / 2}
$$

From the properties of $E_{i}(\cdot)$ above, we have that $\|S(\lambda)\| \leq N M$ almost everywhere. Since for $x \in H(\lambda),\left\|S(\lambda)_{x}\right\|^{2}=\left\langle S(\lambda)^{2} x, x\right\rangle=\sum_{i=1}^{N}\left\|E_{i}^{*}(\lambda) x\right\|^{2}$ and $\|x\|=\left\|\Sigma E_{i}^{*}(\lambda) x\right\|$ $\leq \sum_{i=1}^{N}\left\|E_{i}^{*}(\lambda) x\right\|$, we have at least one $i$ for which $\left\|E_{i}^{*}(\lambda) x\right\| \geq\|x\| / N$ and hence $\|S(\lambda) x\|^{2} \geq\|x\|^{2} / N^{2}$; therefore $\left\|S(\lambda)^{-1}\right\| \leq N$. Hence $S^{i}=\int \bigoplus S(\lambda) \mu(d \lambda)$ and $S^{-1}=$ $\int \bigoplus S(\lambda)^{-1} \mu(d \lambda)$ exist and to finish the proof we need only show that $S^{-1} A S$ is a binormal operator. To show that, we consider first the operators $S^{-1}(\lambda) A(\lambda) S(\lambda)$. If $P_{i}(\lambda)=S(\lambda)^{-1} E_{i}(\lambda) S(\lambda)$, then $P_{i}^{*}(\lambda)=P_{i}(\lambda), \sum_{i=1}^{N} P_{i}(\lambda)=I(\lambda), P_{i}(\lambda) P_{j}(\lambda)=$ $\delta_{i j} P_{i}(\lambda)$, and thus $S(\lambda)^{-1} A(\lambda) S(\lambda)=\sum_{i=1}^{N} S(\lambda)^{-1} A(\lambda) S(\lambda) P_{i}(\lambda)$. Assume that for $i$, $\left|r_{i}(\lambda)-r_{j}(\lambda)\right|>\delta$ for $i \neq j$, then $S(\lambda)^{-1} A(\lambda) S(\lambda) / P_{i}(\lambda) H(\lambda)=r_{i}(\lambda) I(\lambda) / P_{i}(\lambda) H(\lambda)$ and hence $S(\lambda)^{-1} A(\lambda) S(\lambda) P_{i}(\lambda)=r_{i}(\lambda) P_{i}(\lambda)$, which is certainly normal. If there is an $i^{\prime}$ such that $\left|r_{i}(\lambda)-r_{i^{\prime}}(\lambda)\right|^{i}<\delta$, then $\left(S(\lambda)^{-1} A(\lambda) S(\lambda)\right) P_{i}(\lambda)$ is binormal, since

$$
\left(A(\lambda)-r_{i}(\lambda) I(\lambda)\right)\left(A(\lambda)-r_{i}(\lambda) I(\lambda)\right) / E_{i}(\lambda) H(\lambda)=0
$$


(cf. [3, p. 421]). Therefore almost everywhere $S(\lambda)^{-1} A(\lambda) S(\lambda)$ is the direct sum of binormal operators, hence also binormal and therefore $R\left(S A S^{-1}(\lambda)\right)$ satisfies $(*)$ almost everywhere. The proof is complete since by the operational calculus for decomposable operators it is clear that $R\left(S A S^{-1}\right)$ satisfies $(*)$ whenever $R\left(S A S^{-1}(\lambda)\right)$ does almost everywhere.

In the case that $f^{\prime}(z) \neq 0$ on $r_{0}(A)$, then our theorem is known, as already mentioned, and is due to C. Apostol. However, Apostol's theorem can also be obtained from the method of reduction and that proof follows the proof of Theorem 2 with some modifications and simplifications.

Theorem 3 (C. Apostol). Let $f \in \mathcal{F}_{0}(A)$ for which $f^{\prime}(z) \neq 0$ on $r_{0}(A)$. If $f(A)$ is a normal operator, then $A$ is similar to a normal operator.

Proof. In the proof of Theorem 1, we may now assume that $\left|r_{i}(\lambda)-r_{j}(\lambda)\right|>\delta$, whenever $i \neq j$ since $f^{\prime}(z) \neq 0$ on $r_{0}(A)$. We may define the projections $E_{m}(\cdot)$ as in (2) and instead of (3) we have, for each $m$,

$$
\left(A(\lambda)-r_{m}(\lambda) I(\lambda)\right) E_{m}(\lambda)=0 .
$$

The projections $E_{m}(\lambda)$ can be shown to be essentially bounded by a simplified argument. Let $x \in H(\lambda)$, then

$$
\begin{aligned}
\left\|E_{m}(\lambda) x\right\| & \leq \delta^{-(n-1)} \prod_{i \neq m}\left|r_{m}(\lambda)-r_{i}(\lambda)\right|\left\|E_{m}(\lambda) x\right\| \\
& \leq \delta^{-(n-1)}\left\|\prod_{i \neq m}\left(A(\lambda)-r_{i}(\lambda) I(\lambda)\right) x\right\|
\end{aligned}
$$

by $\left(3^{\prime}\right)$; hence $\left\|E_{m}(\lambda)\right\|$ is essentially bounded. If we define $S(\lambda)$, by equation (6), then $S(\lambda)^{-1} A(\lambda) S(\lambda)=\sum_{i=1}^{N} r_{i}(\lambda) P_{i}(\lambda)$, which is clearly normal. Therefore $S^{-1} A S$ is normal and the theorem is proved.

Whenever $f^{\prime}(z) \neq 0$ on $r_{0}(A)$ and $f$ is $1-1$, then $f^{-1} \in \mathcal{F}_{0}(f(A))$ and $A$ is a function of $f(A)$. This remark was noted by M. Finkelstein and A. Lebow [9] and in particular whenever $f(A)$ is normal, then $A$ is equal to a normal operator. This seemingly trivial remark to $C$. Apostol's theorem has an interesting analogous statement and conclusion with respect to Theorem 2 . The condition that $f$ is $1-1$ is replaced by the condition that $f(z)-\zeta$ has at most two solutions, counted to multiplicity, and, as in the discussion above, we may replace "similar to" with "equal to" in the conclusion.

Theorem 4. Let $A$ be an operator and $f$ be a function in $\mathcal{F}_{0}(A)$ so that $f(A)$ is normal. If for each $\zeta$ in $r_{0}(A)$, the equation $f(z)-f(\zeta)$ bas at most two roots in $r_{0}(A)$, counted to their multiplicity, then $A$ is binormal. 
Proof. Let $A=\int \bigoplus A(\lambda) \mu(d \lambda)$ be the primary decomposition of $A$ on $H=$ $\int_{\mathbf{\Lambda}} \bigoplus H(\lambda) \mu(d \lambda)$. As before, we have that the operator $f(A)$ is decomposed as $f(A)$ $=\int \bigoplus f(A(\lambda)) d \mu(\lambda)$, where $f$ is an analytic function on $\sigma(A(\lambda))$ for almost all $\lambda$. By the Putnam-Fugledge theorem, $N$ commutes with $A^{*}$ and hence $N$ belongs to the center of the von Neumann algebra generated by $A$. Therefore, $N$ is also decomposable and $N=\int \bigoplus g(\lambda) I(\lambda) \mu(d \lambda)$, where $g$ is a Borel function. For almost all $\lambda, \sigma(A(\lambda)) \subset r_{0}(A)$, so that almost everywhere, we have $f(A(\lambda))-g(\lambda) I(\lambda)=0$ and by our hypothesis, there are complex numbers $\alpha_{1}, \alpha_{2}$, in $\sigma(A(\lambda))$ and depending on $\lambda$, so that $f(z)-g(\lambda)=\left(z-\alpha_{1}\right)\left(z-\alpha_{2}\right) b(z)$, where $b(z)$ is an analytic function in $\mathfrak{F}(A(\lambda))$ without zeros on $\sigma(A(\lambda))$. Thus $b(A(\lambda))$ is invertible and therefore we have shown that $\left(A(\lambda)-\alpha_{1} I(\lambda)\right)\left(A(\lambda)-\alpha_{2} I(\lambda)\right)=0$. Hence for almost all $\lambda, A(\lambda)$ is binormal since it satisfies a second degree monic polynomial. By the operational properties of decomposable operators, it is clear that if $A_{1}, \cdots, A_{4}$ are in $R(A)$ and $A_{1}(\lambda), \ldots, A_{4}(\lambda)$ satisfy condition $(*) \mu$-a.e., then so do $A_{1}$, $\cdots, A_{4}$. Therefore $A$ is a binormal operator on $H$.

Remark 1. By the Local Mapping Theorem of analytic functions, we can show that $\alpha_{1}$ and $\alpha_{2}$ can be chosen in a Borel measurable way. Thus if we let $N_{1}=$ $\int_{\Lambda} \bigoplus\left(\alpha_{1}(\lambda)+\alpha_{2}(\lambda)\right) I(\lambda) \mu(d \lambda)$ and $N_{2}=\int_{\Lambda} \bigoplus \alpha_{1}(\lambda) \alpha_{2}(\lambda) I(\lambda) \mu(d \lambda)$, then $A^{2}+$ $N_{1} A+N_{2}=0$ and $N_{1}$ and $N_{2}$ are operators in the center of $A$. It follows that this condition is equivalent to $A$ being a binormal operator.

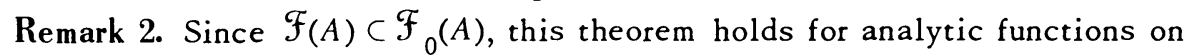
a domain containing $\sigma(A)$.

The reduction methods of this section are clearly not restricted to functions with $f^{\prime \prime}(z) \neq 0$; however there do not exist structure theorems for algebraic operators of degree higher than two which are as complete as that known for binormal operators.

\section{REFERENCES}

1. C. Apostol, On the roots of spectral operator-valued analytic functions, Rev. Roumaine Math. Pures Appl. 13 (1968), 587-589. MR 38 \#2619.

2. H. Behncke, The structure of certain nonnormal operators. II, Indiana Univ。Math。 J. 22 (1972), 301-308.

3. A. Brown, Unitary equivalence of binormal operators, Amer. J. Math. 76 (1954), 414-434. MR 15, 967.

4. T. R. Chow, The spectral radius of a direct integral of operators, Proc. Amer. Math. Soc. 26 (1970), 593-597.

5. - A spectral theory for direct integrals of operators, Math. Ann. 188 (1970), 285-303. MR $42 \# 3598$.

6. J. Dixmier, Les algèbres d'opérateurs dans l'espace Hilbertien, Gauthier-Villars, Paris, 1964.

7. N. Dunford, A spectral theory for certain operators on a direct sum of Hilbert spaces, Math. Ann. 162 (1965/66), 294-330. MR 32 \#182. 
8. N. Dunford and J. T. Schwartz, Linear operators. I, II, III, Interscience, New York, 1958, 1963, 1971. MR 22 \#8302; MR $32 \# 6181$.

9. M. Finkelstein and A. Lebow, A note on "Nth roots of operators", Proc. Amer. Math. Soc. 21 (1969), 250. MR $39 \# 3338$.

10. S. Foguel, Algebraic functions of normal operators, Israel J. Math. 6 (1968), 199201. MR $38 \# 1550$.

11. P. R. Halmos, A Hilbert space problem book, Van Nostrand, Princeton, N. J., 1967. MR 34 \#8178.

12. S. Kurepa, On n-th roots of normal operators, Math. Z. 78 (1962), 285-292. MR $25 \# 1448$.

13. E. R. Lorch, The theory of analytic functions in normed Abelian vector rings, Trans. Amer. Math. Soc. 54 (1943), 414-425. MR 5, 100.

14. J. von Neumann, On rings of operators. Reduction theory, Ann. of Math. (2) 50 (1949), 401-485. MR 10, 548.

15. H. Radjavi and P. Rosenthal, On roots of normal operators, J. Math. Anal. Appl. 34 (1971), 653-664. MR 43 \#3829.

16. J. Schwartz, W*-algebras, Gordon and Breach, New York, 1967. MR 38 \#547.

17. J. G. Stampfli, Roots of scalar operators, Proc. Amer. Math. Soc. 13 (1962), 796798. MR $25 \# 4362$.

DEPARTMENT OF MATHEMATICS, UNIVERSITY OF HAWAII, HONOLULU, HAWAII 96822 